\title{
LA PROBLÉMATIQUE DE LA DÉFINITION DU MOT ABRÉVIATION - DIFFÉRENTS PROCÉDÉS DE CRÉATION DE MOTS NOUVEAUX PAR L'ABRÉVIATION
}

\author{
Radka Fridrichová
}

\begin{abstract}
A wide variety of abbreviations are now present in our reading material and everyday speech. The trend has been incorporated into contemporary French. Time and space is vital. This process takes several different forms which can result in different meanings. The paper covers four different types of abbreviations in this article: initials, acronym, general abbreviations, and truncation.

Keywords: abbreviations; abbreviativ; acronym; initial letter; truncation.

Résumé : Les différentes formes d'abrégé sont présentes au quotidien au travers de nos lectures et de nos conversations. Cette tendance est bien à suivre dans le français contemporain. L'économie de l'espace et du temps est une préoccupation importante. Mais, ce procédé prend des formes diverses et il peut posséder plusieurs définitions. L'article présente quatre différentes formes de raccourci : le sigle, l'acronyme, l'abréviation graphique et la troncation.

Mots clés : abréviation ; abrégé ; acronyme ; sigle ; troncation.
\end{abstract}

\section{Introduction}

L'abréviation est de nos jours un phénomène linguistique très répandu. Le français contemporain est sursaturé par les abréviations. On les trouve partout, dans les journaux, dans les magazines, dans le langage populaire, technique, scientifique, économique etc. Il y a une forte tendance à abréger les mots qui possèdent plus de deux syllabes et par ce procédé, se produisent ensuite des mots nouveaux.

Le mot abréviation peut posséder plusieurs définitions. Pour cette raison, avant de commencer notre analyse de ce domaine d'abrègement, il est nécessaire de définir ce terme. En effet, sous ce mot il est possible de découvrir plusieurs sens qui sont proposés par différents auteurs et qui ont évolué pendant des années.

\section{2. À la recherche d'une définition}

Certains grammairiens prétendent que pour s'occuper d'un problème, il n'est pas nécessaire de donner sa définition, nous ne partageons pas cette opinion et nous considérons que pour pouvoir traiter un problème, il faut d'abord définir le sens même de la notion 
pour éviter des confusions éventuelles. Avant d'étudier n'importe quelle problématique en détail, il est essentiel de présenter au moins une définition générale qui permet de déterminer un certain point de départ. En premier lieu, concernant l'abréviation, nous pouvons constater que l'abréviation est une méthode de création de mots nouveaux. En deuxième lieu, essayons de découvrir et de présenter certains avis et définitions des linguistes qui nous semblent les plus intéressants.

La grammaire d'aujourd'hui : guide alphabétique de linguistique française de Michel Arrivé nous apprend que «l'abréviation est l'une des procédures qui concourent à la néologie lexicale. Elle consiste à manifester une unité linguistique par un signifiant qui, amputé d'un ou plusieurs éléments, conserve le signifié de l'unité de départ. L'abréviation se présente sous des formes diverses. » Il comprend alors sous le nom de l'abréviation les phénomènes comme le sigle, la troncation ou l'ellipse (Arrivé et al., $1986: 17$ ).

L'origine et le sens des mots de Béchade travaille également avec l'abréviation au sens large, c'est-à-dire avec le procédé de création de mots nouveaux et définit la siglaison ainsi que la troncation comme des variantes de l'abréviation. Selon lui, la troncation est "un phénomène qui procède par raccourcissement d'un mot et qui est également appelé troncation » (Béchade, 1994 : 70). Nous pouvons apercevoir qu'il préfère le terme de raccourcissement pour désigner l'abréviation. Dans un autre ouvrage, la Phonétique et morphologie du français moderne et contemporain, H.-D. Béchade emploie le terme : le mot raccourci (Béchade, 1992 : 172).

Ces définitions sont assez convaincantes parce qu'elles utilisent l'abréviation comme l'ensemble des procédés abréviatifs. Malheureusement, elles ne sont pas assez profondes et ne donnent des explications plus claires.

Certains grammairiens préfèrent le terme d'abrègement pour désigner l'abréviation, mais ils éliminent de cette définition les sigles. Ils les considèrent comme un autre procédé.

Citons à titre d'exemple André Martinet qui comprend sous le nom de l'abréviation celui de l'abrègement, et dans La grammaire fonctionnelle $d u$ français, il opère essentiellement avec deux façons de raccourcissement : l'abrègement et le sigle (Martinet, 1979: 265). En effet, l'abrègement est considéré comme procédé de création par l'abréviation, mais le sigle est déjà le résultat du procédé de siglaison.

Pareillement, d'après les auteurs de La grammaire méthodique du français, la langue française emploie deux autres procédés qui enrichissent le vocabulaire : le sigle et l'abréviation. De plus, ils affirment que l'abréviation «donne lieu à la troncation des mots longs " et le mot troncation est en gras pour souligner ce cas particulier de l'abréviation : «l'abréviation constitue une réduction du signifiant d'un mot, le signifié restant en principe inchangé. Elle donne lieu à la troncation des mots longs (plus de trois syllabes), le plus souvent retranchement d'une ou plusieurs syllabes finales; deux ou trois sont conservées, parfois une seule» (Riegel et al., 1994 : 551-552).

Aïno Niklas-Salminen dans sa Lexicologie utilise bien le terme de siglaison et distingue deux procédés d'abrégé : l'abréviation et la siglaison. Il affirme que l'abréviation peut se représenter sous des formes diverses dont la troncation fait partie. De plus, selon lui, "les abréviations sont souvent associées au langage parlé plutôt négligé, les sigles, ..., semblent caractériser avant tout la langue standard » (Niklas-Salminen, 1997:80).

À l'inverse, La grammaire 1. Phonologie, morphologie, lexicologie définit la siglaison comme une forme particulière de l'abréviation (Gardes-Tamine, 1990 : 81) ce qui est une 
affirmation fausse parce que la siglaison est le procédé de la création par l'abréviation qui donne naissance aux sigles et aux acronymes.

Il y a des linguistes dont la définition de l'abréviation est tellement vague qu'il est vraiment difficile de découvrir ce qu'ils entendent par cette notion. Les auteurs de Notre langue française : la grammaire, distinguent d'une part les sigles ou abréviations et d'autre part les abrègements "qu'il ne faut pas confondre avec l'abréviation, paraissent être un tic de langage propre à notre époque » et ils ajoutent que «ce mode de formation - on devrait dire de déformation - est particulièrement répandu dans l'argot estudiantin» (Blois ; Bar, 1975 : 30). Cela démontre que J. Blois et $\mathrm{M}$. Bar comprennent sous l'abrègement un phénomène qui déforme la langue au lieu de la former. En effet, les abrègements n'enrichissent pas le français au niveau de l'esthétique, mais au contraire, ils le dégradent. ${ }^{1}$

Les définitions qui vont un peu plus loin dans leurs distinctions et qui sont assez convaincantes, sont présentées dans Le Bon Usage. Maurice Grevisse sépare d'une manière très stricte la réduction et l'abréviation. Il nous apprend que "l'abréviation est un procédé graphique consistant à écrire un mot en n'utilisant qu'une partie de ses lettres: M. pour Monsieur; $n^{\circ}$ pour numéro. Il n'y a pas de prononciation particulière pour la forme abrégée, ..., il est donc tout a fait gênant d'employer le mot abréviation pour un autre phénomène, que nous appelons réduction » (Grevisse, 1993 : 138). Il considère l'abréviation et la troncation comme deux procédés différents, selon lui, l'abréviation est un phénomène graphique tandis que la troncation est un phénomène lexical (Grevisse, 1993 : 138, 248). Ensuite, il divise la réduction en réduction de mots, cela veut dire la troncation, et en réduction de locutions et de syntagmes (Grevisse, 1993 : 248-249). En revanche, dans sa définition, il ne distingue pas l'abréviation (procédé de création de mots nouveaux) et l'abréviation graphique (le résultat du procédé abréviatif). C'est pour cette raison que nous trouvons sa théorie, elleaussi, insuffisante.

Nous avons vu que les linguistes français, en définissant l'abréviation, ont malheureusement tendance à confondre le procédé de l'abréviation et le résultat de ce procédé, ce qui est incorrect. Les paragraphes précédents nous ont également démontré que les définitions et compréhensions de ces procédés sont parfois très diverses et nous n'avons accepté aucune définition sans réserves.

Il paraît qu'elles sont toutes justifiables sauf celles qui gardent la définition traditionnelle. La dernière comprend sous le sigle une abréviation réduite aux initiales. Cela peut, selon J.-P. Lacroux, être confus parce que d'après ces définitions, l'abréviation $p$. (page) serait en fait un sigle ce qui est une estimation fausse.

À ce propos, il nous semble important de présenter notre propre définition et d'illustrer en profondeur la problématique de l'abréviation. Sous l'abréviation, nous comprendrons, au sens large, l'ensemble des résultats abréviatifs qui seront également appelés abrègements, raccourcissements, abrégés, raccourcis ou mots abrégés/raccourcis. Au sens restreint, nous distinguerons trois procédés d'abrégé (procédé d'abrègement, l'acronymie et la siglaison) qui permettent la naissance de quatre principales formes de raccourci: les sigles, les acronymes ${ }^{2}$, les abréviations (comprenons les abréviations graphiques) et les troncations. L'abréviation est un raccourci uniquement graphique, le sigle est une réduction graphique (aux initiales) affectant aussi la réduction orale (si les lettres sont épelées),

\footnotetext{
1 Cette opinion prend sa source de la date de parution de cet ouvrage, en effet, le français n'était pas d'une manière si profonde envahie par le langage qui s'appuie sur la brièveté.

2 Les acronymes peuvent naître par acronymie et siglaison.
} 
l'acronyme est un mot abrégé aux initiales ou aux premières lettres, celles-ci étant ensuite prononcées comme un seul mot. Enfin, la troncation est une réduction orale (suppression des syllabes au début ou à la fin du mot) qui se répercute sur l'écrit.

Le tableau suivant récapitule les procédés et les résultats de mots nouveaux par l'abréviation: ${ }^{3}$

\begin{tabular}{|c|c|c|c|c|}
\hline & \multirow{2}{*}{$\begin{array}{l}\text { Lieu } \\
\text { de création }\end{array}$} & \multicolumn{3}{|c|}{ Procédés de création de mots nouveaux par l'abréviation } \\
\hline & & procédé d'abrègement & acronymie & siglaison \\
\hline \multirow{2}{*}{$\begin{array}{l}\text { Résultats des pro- } \\
\text { cédés de création }\end{array}$} & oral & troncation & & \\
\hline & écrit & $\frac{\text { abréviations }}{\text { (graphiques) }}$ & $\underline{\text { acronymes }}$ & $\begin{array}{l}\text { sigles, } \\
\underline{\text { acronymes }}\end{array}$ \\
\hline
\end{tabular}

Selon notre tableau, nous recevrons trois procédés qui mènent à quatre types de création, le procédé $\mathrm{d}$ 'abrègement cause la troncation et les abréviations graphiques (en respectant le lieu de création), l'acronymie forme les acronymes, et la siglaison donne naissance aux sigles et aux acronymes. Notons que Lacroux emploie aussi le mot "acronymisation », qui porte sur le mode de lecture, contrairement à la siglaison qui porte sur la formation, car d'après lui, un acronyme peut être obtenu par la siglaison, il est alors " acronymisé » comme l'OTAN (formé des initiales, mais prononcé comme un mot ordinaire). Lacroux appelle également ce type de sigles sigle acronymique (Lacroux, 2007 : 138).

Enfin, le but commun de toutes ces formes abrégées est de gagner soit de l'espace, soit du temps. Mais en outre, d'après Le Bon usage le but des abréviations n'est que de gagner du temps et de la place, mais il y a aussi des abréviations euphémiques employées à la place des mots qui sont jugés soit grossiers soit inconvenants : "Le vulgaire [à l'époque de la Révolution] avait toujours le mot de $f .$. . [ foutre] à la bouche ou sous la plume (Brunot, Hist., $t$. X, p.228); des abréviations de discrétion destinées aux noms propres et de temps en temps aux dates : J'ai achevé avant hier avec l'aide de M., l'article sur les grandes Compagnies (Gide, Journal, 18 août 1927). » (Grevisse, 1993 : 139).

\section{Principaux procédés de création de mots nouveaux par l'abréviation et leurs résultats}

\subsection{Les abréviations graphiques}

Pour les abréviations proprement dites on emploie l'expression abréviation graphique ou selon H.-D. Béchade abréviation typographique (Béchade, 1992: 172). La méthode d'abréviations est décrite comme "un procédé graphique consistant à écrire un mot en n'utilisant qu'une partie de ses lettres" (Grevisse, 1993 : 138).

Il existe différentes manières d'abréger. Cela dépend tout d'abord d'un choix personnel d'abrègement, mais il est bien possible de suivre les règles générales et de trouver les tendances ou les conventions à respecter. En général, trois types d'abréviations sont à distinguer : L'abrègement se réalise par simple suppression de segments du mot ou des mots à abréger. Ce procédé est effectué soit au début d'un mot, soit au début et à la fin de ce mot, soit à l'intérieur du mot.

\footnotetext{
3 L'ensemble des formes abréviatives sera nommé abrègement et le procès par lequel naissent les troncations et les abréviations sera appelé procédé d'abrègement.
} 
a) L'abréviation se fait le plus souvent par la suppression des dernières lettres d'un mot qu'on coupe après une consonne et avant une voyelle. Le fait qu'il s'agit d'un segment abrégé, est marqué par un point (dit point abréviatif), sauf s'il s'agit d'un point cardinal. Le mot est alors réduit à sa lettre ou ses lettres initiales :

Le mot conserve seulement sa première lettre:

Ex. : M. $=$ Monsieur $;$. $=$ page $; t .=$ tome.

L'abréviation des mots composés, des locutions ou des expressions se fait à l'aide d'un élément d'un mot. La ponctuation entre les mots est en général conservée. ${ }^{4}$

Ex. : c.-à-d. $=c^{\prime} e s t-a ̀-d i r e ; ~ c . c .=$ copie conforme $;$ p. $i .=$ par intérim.

Le mot conserve plusieurs lettres initiales:

Ex. : av. =avenue $;$ réf. $=$ référence $;$ chap. $=$ chapitre $;$ fr. ou franç. $=$ français.

Nous pouvons observer que le mot français s'abrège après une consonne et avant une voyelle, pour cette raison, il est impossible de raccourcir le mot français en fra. ou fran. Ces abréviations se terminent le plus souvent par une consonne. Le point abréviatif tient lieu de la lettre finale. (cf. Grevisse, 1993 : 140).

b) Le mot est réduit à son début et à sa fin. Ce procédé d'abréviation est couramment utilisé dans l'écriture manuscrite. Deux traitements différents du segment droit du mot abrégé se proposent : soit il est réalisé en lettres supérieures (c'est-à-dire les lettres finales sont placées plus haut que les autres et parfois soulignées), soit il est réalisé sur la même ligne. L'abréviation qui se termine par la dernière lettre du mot ne comporte pas ce point final :

Ex. $: D^{r}=$ docteur $; M M$. ou $M^{r s}=$ Messieurs $; M^{m e}=$ Madame $; M^{\text {lle }}=$ Mademoiselle $; M^{g r}=$ Monseigneur $; P^{r}=$ Professeur.

L'écriture des lettres sur la même ligne que l'initiale est possible, mais il faut que la suite de lettres donne un mot imprononçable comme Mgr (Monseigneur), Pr (Professeur); si cette suite de lettres est prononçable, elle risque d'être confondue avec un mot. Pour cette raison, il est préférable d'écrire la fin du mot au-dessus de la ligne. Au lieu de no, Cie et $M e$, on écrit donc $n^{\circ}$ (numéro), C Ce (Compagnie), M (Maître) (cf. Grevisse (1993 : 142). Néanmoins, Cie ou no (ou bien No) sont souvent rédigés de manière incorrecte, sur la même ligne.

Remarques: Il faut distinguer l'écriture anglaise des mots comme Madame ou Monsieur. En effet, la langue anglaise met un point abréviatif même si le mot est réduit à son début et à sa fin, $M r .=$ Mister, Mrs. = Mistress. Mais, il n'existe aucune raison pour appliquer la même règle en français et abréger le mot Monsieur en $M r$.

En revanche, l'abréviation $M^{r}$ avec un $r$ en lettre supérieure était utilisée au XVII ${ }^{e}$ siècle en ancien français (cf. Grevisse, 1993 : 141).

Il est recommandable d'employer cette abréviation si l'on souhaite raccourcir par exemple Monsieur Martin Moulin. L'abrègement serait le suivant: M.M. Moulin, mais cela n'est pas très clair. On peut confondre avec MM. Moulin (Messieurs Moulin). Pour éviter les incompréhensions ou difficultés, nous pouvons écrire $M^{r} M$. Moulin pour qu'il soit évident de qui ou de quoi nous envisageons de parler.

c) L'abréviation est née aussi grâce à l'omission de lettres à l'intérieur d'un mot. C'est une méthode qui est très répandue de nos jours surtout en raison de l'extension des nouvelles technologies et surtout de la communication par SMS. Ce procédé est appelé par

\footnotetext{
${ }_{4}^{4}$ Après la lettre à le point abréviatif ne se révèle pas, parce qu'elle n'est pas abrégée.
} 
certains linguistes abréviation par syncope (cf. notamment Krautgartner, 2003 : 2). Cette omission se fait par trois procédés différents :

- omission du graphème ou : pr (pour), jr (jour), tjrs (toujours) ;

- omission des voyelles nasalisées «on, en, an, in, un » etc. : dc (donc), dt (dont), tps (temps), avt (avant), ds (dans);

- omission de toutes les voyelles et quelques consonnes en plus : $r d v$ (rendez-vous), $p b$ (problème), bsr (bonsoir), ajd (aujourd'hui).

Or, la combinaison de ces procédés est possible. Par exemple, le mot bonjour peut être abrégé en bjr ; cela veut dire que dans la première syllabe on a omis la voyelle nasalisée « on » et au niveau de la deuxième syllabe, nous avons négligé le graphème " ou ». De là viennent la plupart des abréviations textuelles et du chat parce qu'il faut écrire vite et économiser les lettres, c'est-à-dire de la place. Ainsi, l'orthographe n'est qu'approximative et les abréviations répondent souvent à la phonétique du mot.

Ex. : slt a vs ts (salut à vous tous); dsl pr $2 m 1$ (désolé pour demain); C pa gro (ce n'est pas grave); merc bcp a tt (merci beaucoup, à toute à l'heure).

Les Français abrègent les mots les plus courants toujours de la même manière. C'est pour cette raison que nous pouvons voir dans les cahiers scolaires les abréviations telles que : $d s, d c, p r$, tj ou $t t$ (dans, donc, pour, toujours, tout) et tout le monde sait comment décrypter ce qu'ils signifient. Toutefois, les mots plus longs peuvent poser des problèmes et à l'inverse, chacun peut former sa propre abréviation (Faraco, $1997: 40$ ).

\subsection{Les sigles}

En suivant les définitions de J.-P. Lacroux nous voyons que les capitales composant le sigle représentent les lettres initiales des mots qui ne sont pas oralisés sous leur forme pleine mais par épellation (SNCF, [عsensef], Société nationale des chemins de fer français) ou par syllabation, c'est-à-dire en formant des syllabes comme pour un mot ordinaire (UNESCO ${ }^{5}$, [ynesko], United Nations educational, scientific and cultural Organization). Si l'oralisation des sigles se fait par la lecture, nous parlons dans ce cas-là d'acronymes (LAcroux, 2007 : 136-137).

De plus, J.-P. Lacroux précise dans son ouvrage les termes susmentionnés pour leur donner une tentative de clarification:

Acronyme (initiales) : OTAN (Organisation du traité de l'Atlantique Nord). ${ }^{6}$

Acronyme (syllabique ou pseudosyllabique) : Benelux (Belgique, Nederland, Luxembourg).

Acronyme hybride (initiales et syllabes) : radar (radio detection and ranging).

Sigle pur (épelé) : CGT (Confédération générale du travail).

Sigle hybride : TF1 (Télévision française).

Le phénomène le plus discutable sur les sigles est celui du point abréviatif. Il marque la coupure à l'intérieur d'un abrégé (V.T.T. = vélo tout terrain). Jadis, le point abréviatif s'imposait dans tous les sigles et acronymes. Cet emploi est encore respecté par certains. Pour cette raison-là, on peut souvent remarquer une écriture différente pour un même sigle.

\footnotetext{
5 Le sigle UNESCO a cessé d'être épelé pour être aujourd'hui prononcé.

6 Lacroux dans son ouvrage Ortotypographie parle aussi du sigle acronymique car il est formé comme un sigle, mais il n'est pas épelé, mais lu comme un mot ordinaire, selon lui, un acronyme peut être obtenu par siglaison, néanmoins à l'inverse ce procédé ne fonctionne pas.
} 
L'exemple de PDG (Président-directeur général) ou bien P.D.G, P.-D.-G., P.-D.G. et P-DG illustre cette affirmation. Il est également possible de rencontrer $p d g$ ou bien pédégé, ils représentent un changement dans l'écriture de ce sigle au cours des années. Il suffit de lire des articles ou consulter Internet et on découvrira des variantes de l'écriture de ce sigle.

Les sigles désignent le plus souvent des sociétés, des partis politiques, des organismes. Certains sigles peuvent aussi indiquer un nom de pays dont la dénomination complète serait trop longue et compliquée : les USA = United States of America, UK = United Kingdom. D'autres relèvent des noms communs: HLM = habitation à loyer modéré, PME $=$ petites et moyennes entreprises. Le plus souvent, ce sont les substantifs qui sont abrégés, mais les sigles peuvent parfois équivaloir aux adjectifs: k.o. $=$ knock-out, ou parfois à des phrases, surtout dans le langage du chat, des messages textuels et des méls pour économiser de nouveau l'espace et le temps:

Ex. : svp = s'il vous plaît $m d r=$ mort de rire $;$ tob $=$ tout va bien; atd $=\grave{a}$ ta disposition $; P Q T$ = plus que toi.

Certains sigles courants entraînent la formation des dérivés: cégétiste $=$ membre de la CGT ou érémiste = bénéficiaire du RMI (revenu minimum d'insertion). Les sigles qui sont de notoriété sont parfois écrits tels qu'on les prononce. Il s'agit ainsi des noms communs qui s'accordent en genre et en nombre : cédérom $=C D$ (compact disc), pédégé $=P D G$, bédé $=B D$ (bande dessinée), vécés $=W C$ (water-closet). Les formes telles que bédé, vécés etc. sont les formes populaires formées à partir de la prononciation courante des sigles, cela signifie que la forme phonétique est lexicalisée. Pour le pluriel, la forme populaire adopte un $s$ à la fin du mot siglé.

Les sigles sont invariables et pour cette raison-ci, ils ne prennent pas, en général, de marque du pluriel, contrairement à l'usage anglo-saxon.

\subsection{Les acronymes}

Comme nous l'avons déjà mentionné, c'est premièrement la prononciation qui distingue un sigle d'un acronyme, puis, en général, les acronymes sont privés de point abréviatif. Certains acronymes sont donc fondés à partir de premières lettres de mots qu'ils servent à réduire : OVNI (Objet Volant Non Identifiê). ${ }^{7} \mathrm{D}^{\prime}$ autres sont formés sur les premières syllabes : DIPER (DIrection du PERsonel) et/ou mêlent initiales et syllabes : RIMA (Régiment $d^{\prime}$ Infanterie de MArine). En outre, beaucoup d'entre eux proposent des combinaisons différentes : COFACE (COmpagnie Française d'Assurance pour le Commerce Extérieur) (cf. Béchade, 1992 : 172-173).

Parfois, les majuscules, sauf la première qui est gardée, se changent en minuscules, et forment alors un nouveau mot (UNESCO ou bien Unesco). Remarquons qu'il est possible d'écrire certains acronymes en minuscules et de les percevoir comme une entité sémantique, c'est le cas des mots suivants : laser (light amplification by stimulated emission of radiation), ovni (objet volant non identifie), radar (radio detection and ranging) qui sont deve-

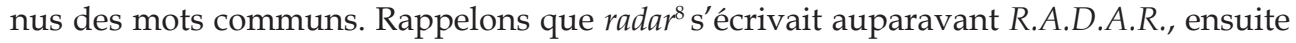
RADAR pour enfin devenir radar.

\footnotetext{
7 Les majuscules montrent le processus de l'abrègement.

8 Cartier souligne que le mot radar a aussi passé dans le langage familier : «Hier j'ai fait la teuf, aujourd'hui je suis au radar. » Cela veut dire dans le français standard : « Hier, j'ai fait la fête, aujourd'hui je suis mort. » (cf. Cartier, 2009 : 169).
} 
À ce propos, il faut souligner que non seulement l'écriture des sigles et des acronymes peut changer, mais aussi la prononciation. En effet, certains usages des acronymes/ sigles, cela veut dire les résultats qui naissent du procédé de siglaison, offrent le choix de prononciation ce qui est le cas d'URSS [yereses] ou [yrs] (Union des républiques socialistes soviétiques) (cf. Dubois, 1965 : 96). Mais, Lacroux mentionne «qu'un sigle pouvant, mais ne devant pas, se lire comme un mot ordinaire n'est pas un acronyme ». À titre d'exemple il cite le sigle OUA (Organisation de l'unité africaine) qui peut se lire comme [oya], mais cette prononciation n'est pas imposée (Lacroux, $2007: 137$ ).

En revanche, certains acronymes mixent les deux possibilités de lecture : DGCID (Direction générale de la coopération internationale et du développement). Les lettres $D$ et $G$ sont épelées et $C I D$ est lu comme un seul mot. Dans le sigle CAPES (Certificat d'aptitude au professorat de l'enseignement du second degré), qui s'oralise en [kapss] et non [kap], les quatre premières lettres sont lues et le $S$ est épelé.

Il faut mentionner que Bartoš affirme que lorsqu'un sigle est formé de trois lettres, il y a deux possibilités de prononciation, et cela dépend de la répartition des consonnes et des voyelles. En général, le sigle est prononcé s'il est formé par consonne - voyelle - consonne tel que par exemple FOM [fom] (Facilité à options multiples). Les sigles qui ont la structure suivante : consonne voyelle - consonne peuvent être prononcés comme un mot ordinaire ou être épelés. Dans les autres cas, les sigles de trois lettres sont épelés selon l'alphabet français (Bartoš, 1987 : 8).

Dans l'usage courant se retrouvent de plus en plus de dérivés formés d'après leurs exemples d'origine. Par cette procédure prennent naissance de nouveaux mots $(O N U=$ Organisation des Nations Unies $\rightarrow$ onusien, qui relève de l'ONU, SMIC = le salaire minimum interprofessionnel de croissance $\rightarrow$ smicard, un bénéficiaire du SMIC, SIDA $\rightarrow$ sidéen, qui est contaminé par SIDA, ENA $\rightarrow$ énarc, étudiant de l'ENA.). Enfin, certaines de ces créations viennent de l'anglais : lol = laughing out loud, asap = as soon as possible.

\subsection{La troncation}

Nous avons déjà mentionné que l'abréviation est un phénomène graphique tandis que la troncation est un phénomène lexical.

De plus, l'abréviation est réduite à l'écrit (nous abrégeons le mot Monsieur en M., mais il est prononcé comme Monsieur, c'est-à-dire comme un mot entier), en revanche, la troncation peut être prononcée sous la forme de son abrègement (il est possible de dire la faculté ou la fac en utilisant la forme raccourcie).

Selon Grevisse la troncation est souvent appelée la réduction. Il résulte que la réduction "est un phénomène lexical donnant naissance à un mot nouveau issu du découpage de plusieurs syllabes finales » (Grevisse, 1993 : 138). Ainsi, en français, certains mots courts sont le résultat de la réduction de mots plus longs.

A la différence des abréviations, les mots tronqués ne prennent pas de point abréviatif. ${ }^{9}$ De plus, beaucoup de mots tronqués appartiennent à la langue familière, populaire et même argotique. Ces formes réduites sont souvent des noms et des adjectifs dont la longueur n'est pas nécessairement excessive. Les mots s'abrègent généralement par suppression de leurs syllabes initiales ou finales et ils se terminent fréquemment par une consonne. Deux différents cas de la troncation sont à distinguer, soit à la droite du mot (apocope), soit à la gauche (aphérèse) (cf. Groud ; Serna, 1996 : XIII).

\footnotetext{
9 Il ne s'agit pas d'une règle grammaticale mais d'une convention de typographie.
} 
La problématique de la définition du mot abréviation...

\subsubsection{Troncation à droite (apocope)}

À partir de trois syllabes les mots sont considérés comme longs, et pour cette raison, les différents modes de raccourcissement sont mis en place. L'apocope est le procédé de troncation le plus souvent utilisé. En effet, c'est un procédé qui retranche les syllabes finales d'un mot. La coupure conserve le premier élément d'un composé qui est d'origine savante ou, dans les autres cas, elle est effectuée après la deuxième ou troisième syllabe ou après une syllabe qui se termine par $o$.

L'apocope efface fréquemment les dernières syllabes, deux ou trois syllabes sont conservées, parfois une seule:

Ex. $: f a c=$ faculté $;$ amphi $=$ amphithéâtre $;$ ciné $=$ cinéma $;$ bac $=$ baccalauréat .

Le découpage des syllabes n'est pas toujours respecté et identique pour tous les mots. Les mots manif(estation), prof(esseur), fac(ulté) se terminent par la consonne initiale de la deuxième ou parfois de la troisième syllabe du mot qui est raccourci.

Très fréquemment, la troncation isole un mot préfixé à son préfixe :

Ex. : hyper $=$ hypermarché ; super $=$ supercarburant ${ }^{10}$.

En plus, la réduction touche aussi des mots composés savants :

Ex. : cinéma = cinématographe $;$ kilo = kilogramme $;$ moto $=$ motocyclette $;$ stylo = stylographe.

Dans les mots composés, le premier élément représente le mot réduit comme télé(vision), mais le découpage n'est pas toujours exact (météo = météoro-logie).

Remarques: Le mot professeur raccourci comme $P^{r}$ est l'abréviation - au sens restreint, mais professeur comme prof indique la troncation. Il faut veiller à ne pas confondre les termes de troncation et d'abréviation (ici, comprenons abréviation graphique). Le même phénomène se produit en cas de kilogramme, l'abrégé $k g$ est né de l'abréviation, et kilo est la réduction du même mot.

\subsubsection{Les suffixes employés après troncation}

Les mots tronqués avec un $o$ final, sont une procédure plus spécifique qui consiste à ajouter la finale $o$ à certains mots tronqués par apocope, c'est-à-dire, dont les syllabes finales ont été retranchées. Cette finale en $o$ est empruntée aux bases des composés logo-, proto-, expo-, vélo-, labo- etc. et qui a pour origine le suffixe diminutif en -ot. D'où viennent les formations suivantes :

Ex. $:$ alcoolo $=$ alcoolique $;$ apéro $=$ apéritif $;$ prolo $=$ prolétaire $;$ hosto $=$ hôpital $(\mathrm{cf}$. NiklasSalminen, 1997: 81).

D'où aussi la forme féminine de quelques abréviations en $o$ comme directeur $\rightarrow$ dirlo $\rightarrow$ dirlote.

L'homophonie avec le suffixe nominal -ot $t^{11}$ indique la présence des mots populaires et ce suffixe est en effet d'origine diminutive et parfois aussi péjorative.

Ex. : baccalauréat $\rightarrow$ bachot; frère $\rightarrow$ frérot; cul $\rightarrow$ culot.

Ces termes en $-o$ ont une coloration argotique ou familière, et de temps en temps la connotation peut être plutôt positive que négative. La réduction accro (avoir une vive

${ }^{10}$ Ces préfixes ou monèmes qui sont libérés par raccourcissement et par ce procédé-là ils ont obtenu une autonomie, ex. : extra, ultra, super, mini. Ils peuvent être utilisés comme des noms : J'ai fait plein de super, des adjectifs : Cette galette est extra ou des adverbes: C'est super bon et finalement ils peuvent être réemployés comme des affixes. (cf. Martinet, $1997: 265$ ).

${ }^{11}$ Ce suffixe est d'origine latine -ottum, la forme féminine en français est -otte, il peut indiquer des diminutifs, une nuance affective ou seulement une relation comme dans les mots culotte (cf. Grevisse, 1993 : 217). 
inclination pour qqch, aimer faire qqch) qui vient du mot accroché est en fait plus positive que sa forme entière. Au contraire, les mots comme intello (intellectuel), bolcho (bolchevique), écolo(écologique) ou catho (catholique) ont une connotation péjorative.

La plupart des mots tronqués sont formés par la simple coupure du mot entier ou encore par l'ajout du suffixe $o$. Mais, dans le français il y a d'autres suffixes qui ont une connotation familière, appelés pseudo-suffixes. Martin Riegel les appelle suffixes vides qui servent " pour l'œil ». Ils s'ajoutent sur des formes tronquées, et il s'agit des suffixes comme -ar, -oche, -lo, -os (Riegel, 1997 : 552). En revanche, J.-P. Goudailler parle de resuffixation après troncation. "Il s'agit là aussi d'un procédé formel typiquement argotique ; l'argot traditionnel est bien connu pour ses resuffixations, entre autres, en -asse, -os, -ard. ». (Goudailler, $2001: 28$ ). Les deux auteurs sont d'accord pour dire qu'il s'agit d'un procédé formel ("pour l'œil ») qui est en plus d'une tradition argotique.

Ex. : polar $=$ roman policier $;$ cinéma $=$ cinoche $;$ valise $=$ valdoche $;$ américain $=$ amerlo ; matériel $=$ matos. ${ }^{12}$.

Mais, dans ce cas-là, nous ne pouvons pas parler de vraie économie d'espace.

Dans certains cas le mot peut être verlanisé ${ }^{13}$, ensuite tronqué et à la fin nous ajoutons encore un suffixe. C'est par exemple le cas du mot rabzouille, le mot d'origine est les arabes, il est verlanisé en rabza et ensuite nous procédons à la resuffixation en -ouille, c'est-à-dire rabzouille.

\subsubsection{Troncation à gauche (aphérèse)}

L'aphérèse consiste en la chute au début d'un mot de phonèmes, de lettres ou de syllabes. Ce type de réduction est souvent utilisé dans la langue parlée ou dans certains contextes à l'écrit où il représente les expressions familières. Il apparaît également dans le langage enfantin, argotique ou dans les diminutifs des prénoms. Enfin, il faut constater que cette réduction est plus rare que l'apocope.

Il est à souligner que ce type de troncation est beaucoup plus cryptique que l'apocope parce que c'est la première syllabe qui apporte le plus d'informations au niveau sémantique.

Ex. : Bus $=$ autobus $;$ cipal $=$ principal $;$ pitaine $=$ capitaine $;$ Toine $=$ Antoine .

Parfois, une combinaison de l'apocope et de l'aphérèse pour un même mot est possible : maréchal des logis $\rightarrow$ margis, boulevard Saint-Michel $\rightarrow$ Boul'mich, festival de cinéma $\rightarrow$ cinéstival. (cf. Grevisse, $1993: 248$ ).

En outre, le phénomène de l'aphérèse peut être suivi par le procédé de redoublement. J.-P. Goudaillier l'explique comme "un procédé formel de formation des mots qui relève essentiellement de la fonction ludique du langage » (Goudailler, $2001: 28$ ). Cette forme est surtout utilisée par les enfants.

Ex. $:$ dic $=$ dicdic $\left(\right.$ indicateur de police) $;$ fan $=$ fanfan $($ enfant $) ;$ zon $=$ zonzon $($ prison $) .{ }^{14}$

${ }_{12}$ Dans les mots qui finissent en suffixe -os, le $s$ final est prononcé.

${ }^{13}$ Le verlan est une langue cryptique, il consiste à inverser les syllabes dans un même mot. Il était d'abord utilisé par les jeunes immigrés pour se ensuite répandre parmi les adolescents de tous les milieux.

${ }_{14}$ Pour garder la prononciation de la lettre $z$ [zed], il fallait changer la lettre initiale du mot tronqué de $s$ en $z$. Sinon, on prononcerait [sõ], au lieu de [zõ]. 
La problématique de la définition du mot abréviation...

\section{Conclusion}

Le français contemporain est sursaturé par les différents types d'abrégé des mots. Ils sont présents au quotidien au travers de nos lectures et de nos conversations. Le terme d'abréviation semble facile à définir. Nous avons pu voir que sa définition est pourtant complexe. Mais, tous les procédés de raccourcissement que nous avons traité ont un trait en commun ; ils cherchent premièrement à économiser le temps ainsi que l'espace.

\section{Bibliographie}

Arrivé, Michel et al. (1986), La grammaire d'aujourd'hui: guide alphabétique de linguistique française, Paris : Flammarion, 1986.

BArToš, Jozef (1987), Francúzske skratky, Bratislava.

BAUssier, Sylvie (2007), Les sigles à la con, Paris : Mots \& Cie.

BÉCHADE, Hervé-D. (1992), Phonétique et morphologie du français moderne et contemporain, Paris : PUF.

BÉCHADE, Hervé-D. (1994), L'origine et le sens des mots, Paris : PUF.

BloIs, Jacques ; BAR, Marc, (1975), Notre langue française, Paris : Didier.

CAlvet, Jean (1980), Les sigles. Que sais-je ?, Paris : PUF.

CAmus, Jean-Christophe (1997), Zappe ton sigle, Paris : Michel Lafon.

CARTiER, Patrice (2009), Le langage des sigles, Paris : De La Martinière.

DAuzet, Albert et al. (1971), Nouveau dictionnaire étymologique et historique, Paris: Larousse.

Doppagne, Albert (1991), Majuscules, abréviations, symboles et sigles, Pour une toilette parfaite du texte, Paris : Duculot.

DuвоIS, Jean (1965), Grammaire structurelle du français : nom et pronom, Paris: Larousse.

FARACO, Martine (1997), «Technique de prise de notes en français spécialisé », in : Le Français dans le monde, 287.

Gardes-TAmine, Joëlle (1990), La Grammaire, 1. Phonologie, morphologie, lexicologie: méthode et exercices corrigés, Paris : Armand Colin.

GAUDIN, François ; GuESPIN, Louis (2000), Initiation à la lexicologie française. Bruxelles : Duculot.

GoudaILLER, Jean-Pierre (2001), Comment tu tchatches, Paris : Maisonneuve et Larose.

GoudAILLER, Jean-Pierre (2002), « De l'argot traditionnel au français contemporain des cités », La Linguistique 38, 1.

Grevisse, Maurice (1993), Le Bon Usage, Paris : Duculot (13eéd. refondue par Goose, André).

Groud, Claudette ; SERnA, Nicole (1996), De abdom à zoo, Regards sur la troncation en français contemporain, Paris : Didier Érudition.

Krautgartner, Klare (2003), "Techniques d'abréviation dans les webchats francophones ", in : Linguistik online, 15, 3. Disponible sur http://www. linguistik-online.de/15_03/krautgartner.pdf, consulté le 17/12/2010.

Lacroux, Jean-Pierre (2007), Orthotypographie, Custines: Hélio Service. Disponible sur http://creativecommons.org/licenses/by-nc-nd/2.0/fr/, consulté le 11/02/2011.

Lehmann, Alise; Martin-Berthet, Françoise (2003), Introduction à la lexicologie, Paris : Armand Colin.

Martinet, André (1979), Grammaire fonctionnelle du français, Paris : Didier. 
MAUGER, Gaston (1979), Grammaire pratique du français d'aujourd'hui : langue parlée, langue écrite, Paris : Hachette.

MitTERAND, Henri (1963), Les mots français, Paris : PUF.

Mortureux, Marie-Françoise (2008), La lexicologie entre langues et discours, Paris : Armand Colin.

Niklas-Salminen, Aïno (1997), La lexicologie, Paris : Armand Colin.

POlguÈre, Alain (2008), Lexicologie et sémantique lexicale, Notions fondamentales, Montréal : Les Presses de l’Université de Montréal.

RIEGEL, Martin et al. (1994), Grammaire méthodique du français, Paris : PUF.

VAIREL, Hélène (1989), La présentation matérielle d'un manuscrit dactylographié, Poitiers : Nathan.

\author{
Radka Fridrichová \\ Pedagogická fakulta \\ Západočeská univerzita v Plzni \\ Jungmannova 1 \\ Plzeň 30614 \\ RFridrichova@seznam.cz \\ République tchèque
}

